\title{
A novel endoplasmic reticulum stress-induced apoptosis model using tunicamycin in primary cultured neonatal rat cardiomyocytes
}

\author{
MINGZHI SHEN ${ }^{1-3 *}$, LIN WANG $^{1 *}$, XIAOWANG GUO ${ }^{1}$, QIAO XUE $^{2}$, \\ CONG HUO $^{1}$, XING LI ${ }^{1}$, LI FAN $^{3}$ and XIAOMING WANG ${ }^{1}$ \\ ${ }^{1}$ Department of Geriatrics, Xijing Hospital, Fourth Military Medical University, Xi'an, Shaanxi 710032; \\ ${ }^{2}$ Department of Cardiology, Hainan Branch of PLA General Hospital, Sanya, Hainan 572013; \\ ${ }^{3}$ Department of Geriatric Cardiology, Chinese PLA General Hospital, Beijing 100853, P.R. China
}

Received August 8, 2014; Accepted May 6, 2015

DOI: $10.3892 / \mathrm{mmr} .2015 .4040$

\begin{abstract}
Endoplasmic reticulum (ER) stress is key in the development of cardiovascular diseases. However, there is a lack of a systemic ER stress-induced cardiomyocyte apoptosis model. In the present study, primary cultured neonatal rat cardiomyocytes were exposed to tunicamycin. Cell viability was determined by an MTT assay, and cell damage was detected by a lactose dehydrogenase assay. Flow cytometry was used and the activity of caspase- 3 was analyzed in order to measure apoptosis. Reverse transcription-quantitative polymerase chain reaction and western blotting were used to examine the expression of glucose-regulated protein $78-\mathrm{kDa}$ (GRP78) and C/EBP homologous protein (CHOP). As a result, tunicamycin significantly increased cardiomyocyte injury, which occurred in a time- and concentration-dependent manner. In addition, tunicamycin treatment resulted in apoptosis of cardiomyocytes. Molecularly, tunicamycin $(100 \mathrm{ng} / \mathrm{ml})$ increased the levels of GRP78 and CHOP $6 \mathrm{~h}$ after administration. In addition, GRP78 and CHOP reached maximum mRNA and protein levels $24 \mathrm{~h}$ after administration. In conclusion, the results implicate that the tunicamycin-induced ER stress-induced apoptotic model was successfully constructed in cultured neonatal rat cardiomyocytes. A $100 \mathrm{ng} / \mathrm{ml}$ concentration of tunicamycin was
\end{abstract}

Correspondence to: Professor Xiaoming Wang, Department of Geriatrics, Xijing Hospital, Fourth Military Medical University, 15 Changle West Road, Xi'an, Shaanxi 710032, P.R. China

E-mail: xmwang@fmmu.edu.cn

Professor Li Fan, Department of Geriatric Cardiology, Chinese PLA General Hospital, 28 Fuxing Road, Beijing 100853, P.R. China E-mail: fanli301@sina.com

*Contributed equally

Key words: tunicamycin, endoplasmic reticulum stress, apoptosis, cardiomyocyte, GRP78, C/EBP homologous protein selected, and MTT, LDH release and flow cytometry assay was at $72 \mathrm{~h}$. In addition, GRP78 and GRP94 were detected $24 \mathrm{~h}$ following administration. The results of the present study indicate a novel experimental basis for the investigation of ERS-induced cardiac apoptosis.

\section{Introduction}

The endoplasmic reticulum (ER) is one of the largest cellular organelles and has diverse functions, such as regulating the folding of membrane proteins and secretory proteins (1). Various stimuli, such as ischemia (2), hypoxia (3), heat shock, genetic mutation (4), oxidative stress (5) and elevated protein synthesis could result in ER dysfunction. Stresses that lead to the impairment of ER function are collectively known as ER stress $(6,7)$. ER stress triggers an evolutionarily conserved response termed the unfolded protein response (UPR), an adaptive mechanism that initially promotes organelle recovery (8). In response to ER stress, there is significant upregulation of various ER resident chaperones, such as glucose-regulated protein 94-kDa (GRP94) and glucose-regulated protein $78-\mathrm{kDa}$ (GRP78) that inhibit protein synthesis and activate protein degradation (9-10). When ER stress is excessive and/or prolonged however, apoptotic cell death is triggered by transcriptional induction of C/EBP homologous protein (CHOP) and/or by the activation of c-JUN NH2-terminal kinase (JNK), and/or caspase-12-dependent pathways (11).

Accumulating evidence demonstrates that ER stress-induced apoptosis is the key contributor to cell loss in the pathogenesis of a series of cardiovascular diseases, such as ischemia/reperfusion heart diseases $(12,13)$, atherosclerosis $(6,14,15)$, acute coronary syndrome (16), myocardial infarction $(17,18)$ and heart failure $(19,20)$. Tunicamycin acts as a highly specific ER stress inducer by inhibiting N-linked glycosylation of protein. Since there is a lack of a systemic ER stress-induced apoptotic model in cardiomyocytes, the present study constructed an endoplasmic reticulum stress-induced apoptotic model using tunicamycin in primary cultured rat neonatal cardiomyocytes. The optimal treatment time and 
concentration of tunicamycin in cardiomyocytes was investigated. Cell viability was detected using an MTT assay and cell damage was observed using an LDH release assay. Apoptosis was measured using a flow cytometry assay and determining the activity of caspase-3. Finally, the expression of ER stress markers, including GRP78 and CHOP was induced by tunicamycin at different time points in cardiomyocytes.

\section{Materials and methods}

Reagents and antibodies. Tunicamycin, collagenase I, 3-(4,5-dimethylthiazol-2-yl)-2,5-diphenyltetrazolium bromide (MTT), lactate dehydrogenase (LDH), dimethyl sulfoxide (DMSO), 5-bromo-2-deoxyuridine (BrdU) and trypsin were purchased from Sigma-Aldrich (St. Louis, MO, USA). Tunicamycin was dissolved in DMSO. Dulbecco's modified Eagle's medium (DMEM) medium and fetal bovine serum (FBS) were purchased from Gibco (Grand Island, NY, USA). A bicinchoninic acid (BCA) protein assay kit was purchased from Pierce (Rockford, IL, USA). Anti-GRP78 antibody was obtained from Bioworld (St. Louis Park, MN, USA). Anti-CHOP and anti- $\beta$-actin were obtained from Santa Cruz Biotechnology, Inc. (Santa Cruz, CA, USA). Goat anti-rabbit and goat anti-mouse antibodies conjugated to IRDyeTM ${ }^{800}$ (Rockland Inc., IL, USA) were detected using an Odyssey infrared imaging system (LI-COR Inc., Lincoln, NE, USA).

Culture of primary neonatal rat cardiomyocytes. Primary culture of neonatal rat cardiomyocytes was prepared with the use of 53 neonatal Sprague-Dawley rats from the Fourth Military Medical University (Xi'an, China), as described previously (21). In brief, neonatal rat ventricles were digested with collagenase I and cardiomyocytes were purified by $1 \mathrm{~h}$ incubation at $37^{\circ} \mathrm{C}$ in a $5 \% \mathrm{CO}_{2}$ incubator. Cardiomyocytes were cultured in DMEM medium (containing $0.1 \mathrm{mmol} / \mathrm{l}$ BrdU) with $10 \%$ FBS. Cardiomyocytes amounted for $90-95 \%$ of total adherent cells and then were treated with tunicamycin at multiple concentrations $(25,50,100,200$ and $500 \mathrm{ng} / \mathrm{ml})$ and time-points (24, 48,72 and $96 \mathrm{~h}$ ).. All procedures involving animals were conducted in accordance with the Guide for the Care and Use of Laboratory Animals published by the US National Institutes of Health (NIH Publication no. 85-23, revised 1996), and approved by the Fourth Military Medical University Committee on Animal Care (Xi'an, China).

Cell viability determined by an MTT assay. Cell viability was assessed by an MTT assay as described previously (22). Briefly, cardiomyocytes were seeded in 96-well plates at a density of $5 \times 10^{4} /$ well. After tunicamycin administration at the concentrations and durations, described above, MTT solution $(10 \mu 1,5 \mathrm{mg} / \mathrm{ml}$ in PBS) was added to each well and incubated at $37^{\circ} \mathrm{C}$ for $4 \mathrm{~h}$. Then, the medium was replaced by $150 \mu \mathrm{l}$ DMSO per well. The plate was gently shaken for $5 \mathrm{~min}$ to completely dissolve the precipitate. The absorbance was measured at $490 \mathrm{~nm}$ using a microplate reader (Model 680; Bio-Rad, Hercules, CA, USA). Cell viability was expressed as a percentage of the control.

$L D H$ release assay. To determine cardiomyocyte injury, $\mathrm{LDH}$ release in the medium was detected as described previously (21). The LDH release level was expressed as the rate of $\mathrm{LDH}$ released in the medium to total cellular $\mathrm{LDH}$.

Apoptosis analysis by flow cytometry assay. An Annexin V-fluorescein isothiocyanate (FITC) Apoptosis Detection kit (Sigma-Aldrich) was used to detect apoptosis as described previously (23). Following treatment, cardiomyocytes were washed twice with cold PBS and resuspended in binding buffer. FITC-Annexin V and propidium iodide were added according to the manufacturer's instructions. The mixture was incubated for $10 \mathrm{~min}$ in the dark at room temperature and then cellular fluorescence was measured with a FACSscan flow cytometer (Becton Dickinson, Franklin Lakes, NJ, USA). Annexin V labeled with a fluorophore could identify cells in the early stage of apoptosis, and PI was responsible for staining cells in the medium at late stages of apoptosis. The apoptotic rate was calculated as the percentage of Annexin V-positive and PI-negative cells divided by the total number of cells in the gated region.

Reverse transcription-quantitative polymerase chain reaction $(R T-q P C R)$. The cardiomyocytes were exposed to tunicamycin at the corresponding concentrations and time-points, following which the cells were removed through scraping with a cell scratch and the addition of TRIzol to lyse the cells. cDNA synthesis was performed using a QuantiTect Reverse Transcription kit with $1 \mu \mathrm{g}$ total RNA (Takara Bio, Inc., Shanghai, China). PCR was performed on a Bio-Rad system using Power SYBR Green PCR Master mix (Applied Biosystems, Foster City, CA, USA). cDNA was diluted at 1:5 for each reaction and all qPCR performed using SYBR Green. The reaction conditions were $10 \mathrm{~min}$ at $95^{\circ} \mathrm{C}$, and then 40 cycles of $95^{\circ} \mathrm{C}$ for $15 \mathrm{sec}$ and $60^{\circ} \mathrm{C}$ for $1 \mathrm{~min}$. Rat $\beta$-actin was used to normalize sample amplification. The following primer sequences were used: GRP78, forward: 5'-CTACCGGGACGAGGTACTGG-3' and reverse 5'-GGAAAAGGCGGTGAGGACTT-3'; CHOP, forward: 5'-CGGAGTGTACCCAGCACCATCA-3' and reverse 5'-CCСТCTCCTTTGGTCTACCCTCA-3'; and $\beta$-actin. $\beta$-actin, forward 5'-AGAGGGAAATCGTGCGTGAC-3' and reverse 5'-TTCTCCAGGGAGGAAGAGGAT-3'.

Western blot analysis for GRP78 and CHOP. Cardiomyocytes were lysed using radioimmunoprecipitation assay buffer (Beyotime Institute of Biotechnology, Haimen, China) in combination with a protease inhibitor cocktail (Roche Diagnostics, Mannheim, Germany). Electrophoresis and immunoblotting were conducted as described previously (24). In brief, following treatment with tunicamycin, cardiomyocytes were washed three times with ice-cold PBS and isolated. Proteins were extracted from cardiomyocytes and protein concentrations were determined using a BCA protein assay kit. Protein samples were loaded on $12 \%$ SDS-PAGE gels (Beyotime Institute of Biotechnology), and transferred onto nitrocellulose membranes (Pierce Biotechnology, Inc., Rockford, IL, USA). Following blocking with 5\% non-fat milk in TBS containing $0.1 \%$ Tween-20 (TBS-T) for $1 \mathrm{~h}$ at room temperature, protein bands were reacted with rabbit anti-rat GRP78 antibody (dilution, 1:1,000; cat. no. BS1154; Bioworld), rabbit anti-rat CHOP antibody (dilution, 1:500; cat. no. sc-575; Santa Cruz Biotechnology, Inc.) or mouse anti-rat $\beta$-actin 
A

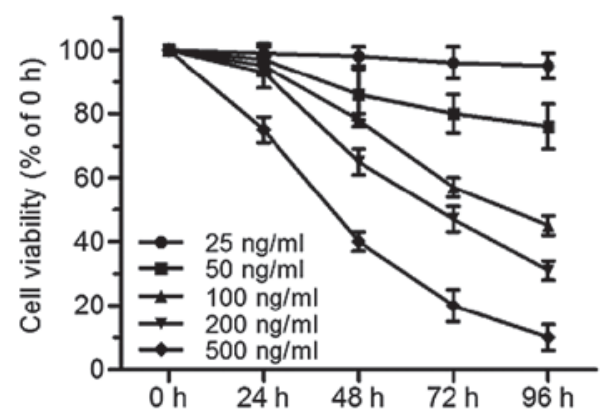

B

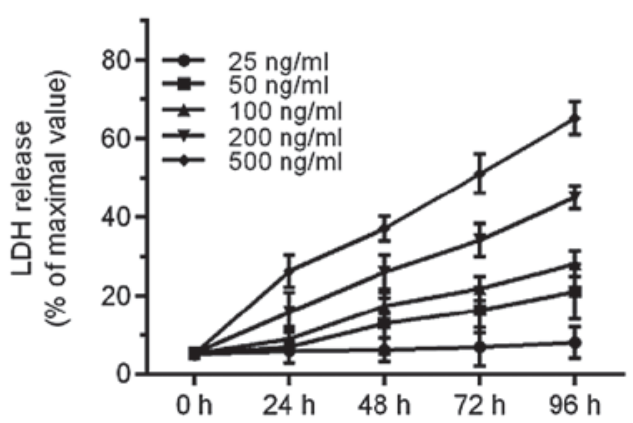

Figure 1. Tunicamycin induces cell injury in primary cultured neonatal rat cardiomyocytes. Cardiomyocytes were treated with tunicamycin $(25,50,100,200$ and $500 \mathrm{ng} / \mathrm{ml})$ for the indicated times (0, 24, 48, 72, $96 \mathrm{~h})$. (A) Cell viability was measured by an 3-(4,5-dimethylthiazol-2-yl)-2,5-diphenyltetrazolium bromide assay and (B) cell injury was analyzed by the LDH release assay. For cell viability, the viable cell number was expressed as a percentage of the 0 h group. For the LDH release assay, all values were compared with the 0 h group. $n=5$. LDH, lactose dehydrogenase.

A
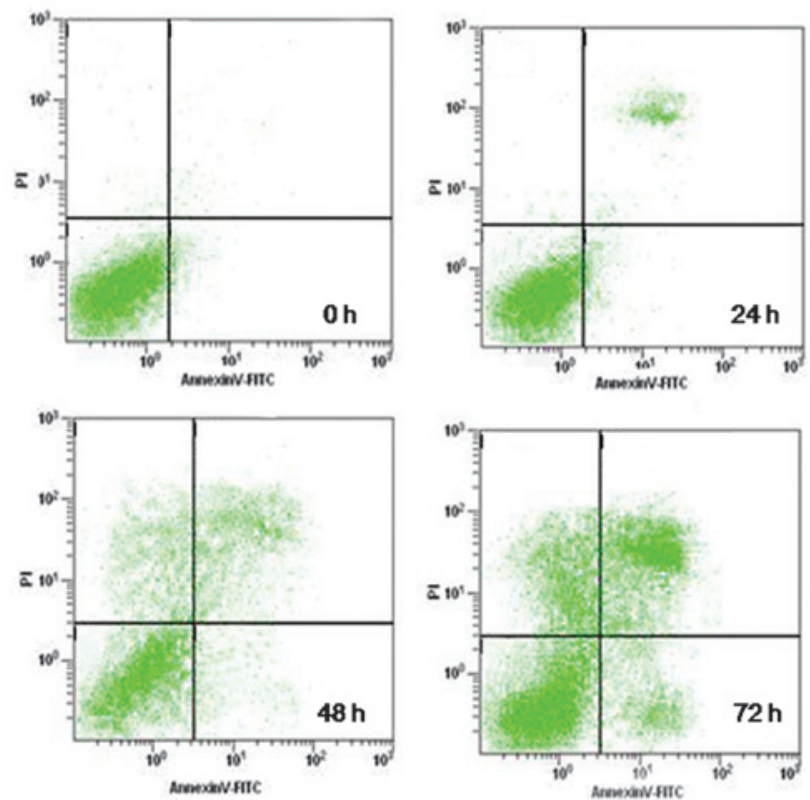

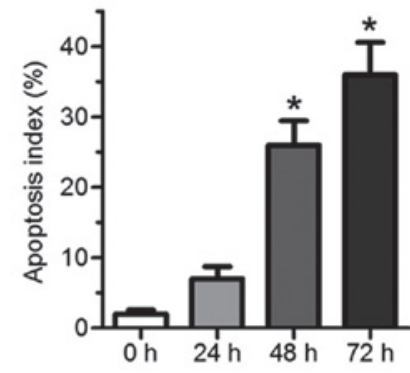

B
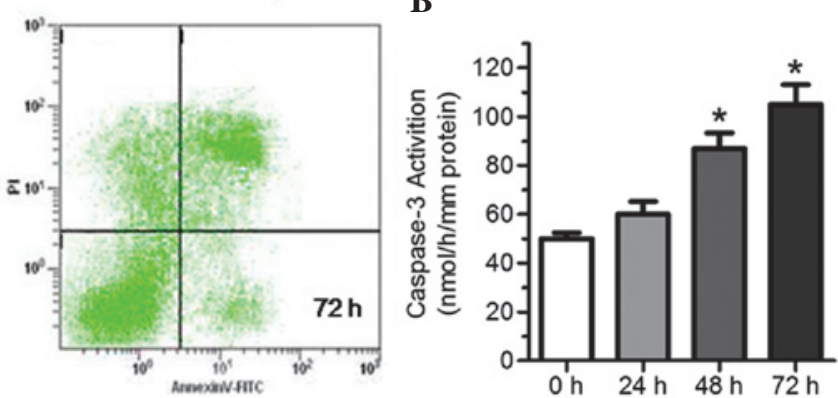

Figure 2. Tunicamycin-induced cell injury manifested as apparent apoptosis in primary cultured neonatal rat cardiomyocytes. Cardiomyocytes were treated with $100 \mathrm{ng} / \mathrm{ml}$ tunicamycin for the indicated times $(0,24,48,72 \mathrm{~h}$ ). (A) Cardiomyocyte apoptosis was detected by flow cytometry. Quantitative analysis of apoptotic-positive cardiomyocytes. (B) The activity of caspase- 3 in cardiomyocytes treated $100 \mathrm{ng} / \mathrm{ml}$ tunicamycin for the indicated times ( $0,24,48,72 \mathrm{~h})$. ${ }^{*} \mathrm{P}<0.05$ vs. $0 \mathrm{~h}, \mathrm{n}=5$.

antibody (dilution, 1:1,000; cat. no. sc-47778; Santa Cruz Biotechnology, Inc) in TBS overnight at $4^{\circ} \mathrm{C}$. Following washing three times with TBST, the membranes were hybridized with goat anti-rabbit or goat anti-mouse DyLIGHT ${ }^{800}$ antibodies for $1 \mathrm{~h}$ at room temperature. Following three washes with TBST, protein bands were visualized on infrared image system (Odyssey; LI-COR Biosciences, Inc., Lincoln, NE, USA). For the densitometry analysis, optical density was measured on the inverted digital images using Image $\mathrm{J}$ 1.46 software.

Statistical analysis. All experiments were performed at least five times. Data are expressed as the mean \pm standard error of the mean. The results were compared by one-way analysis of variance followed by Bonferroni's test. $\mathrm{P}<0.05$ was considered to indicate a statistically significant difference.

\section{Results}

ER stress inducer tunicamycin results in cardiomyocyte injury. An MTT assay and $\mathrm{LDH}$ release assay were used to evaluate cell injury in tunicamycin-treated cardiomyocytes. Tunicamycin, a pharmacological agent inhibiting N-linked protein glycosylation, could be used to experimentally induce ER stress and subsequent cell death. Concentrations of $25-500 \mathrm{ng} / \mathrm{ml}$ tunicamycin were selected. Compared with the control group, $25 \mathrm{ng} / \mathrm{ml}$ tunicamycin for 24-96 h had no effect on cell injury. However, cell viability decreased, and LDH release increased after 48-96 h exposure to tunicamycin at concentrations of 50, 100, 200 and $500 \mathrm{ng} / \mathrm{ml}$ (Fig. 1A and B). Treatment with $100 \mathrm{ng} / \mathrm{ml}$ tunicamycin for $72 \mathrm{~h}$ resulted in a decrease of cell viability to $57.4 \pm 3.2 \%$. These results provide direct evidence 
A

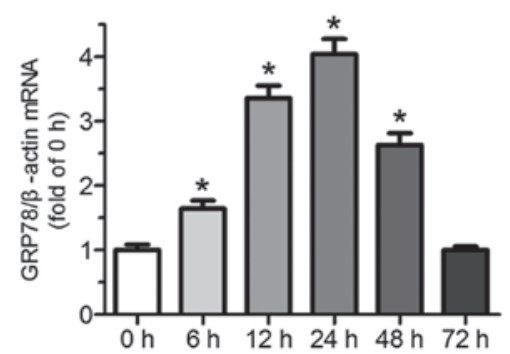

B

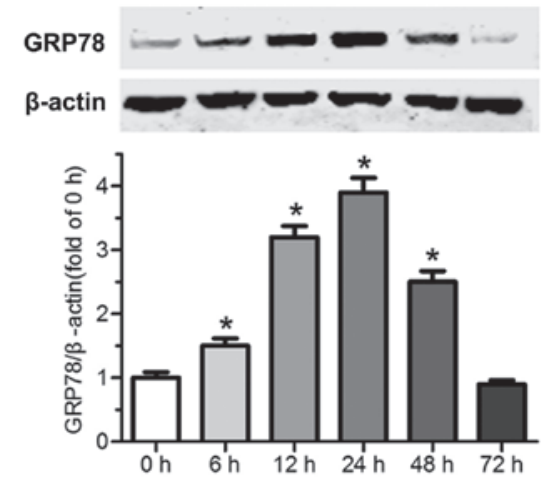

Figure 3. Tunicamycin induced upregulation of the ER chaperone GRP78 in cardiomyocytes. Cardiomyocytes were treated with $100 \mathrm{ng} / \mathrm{ml}$ tunicamycin for the indicated times $(0,6,12,24,48,72 \mathrm{~h})$. (A) The expression of GRP78 mRNA levels was examined by RT-reverse trasncription-quantitative polymerase chain reaction. $\beta$-actin served as an internal control. (B) The expression of GRP78 at protein levels was examined by western blot analysis. $\beta$-actin served as a loading control. ${ }^{*} \mathrm{P}<0.05$ vs. $0 \mathrm{~h}, \mathrm{n}=5$.

A

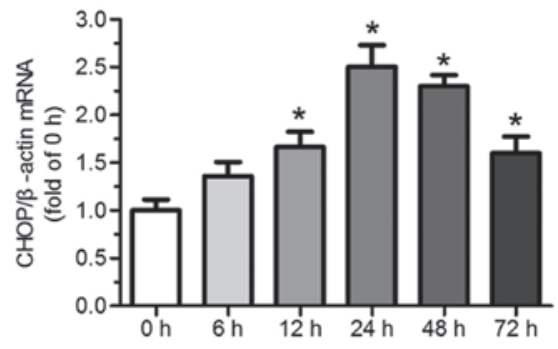

B

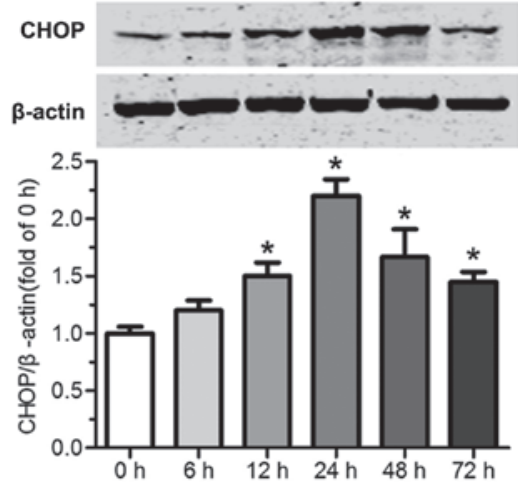

Figure 4. Tunicamycin induced expression of $\mathrm{CHOP}$ in cardiomyocytes. Cardiomyocytes were treated with $100 \mathrm{ng} / \mathrm{ml}$ tunicamycin for the indicated times $(0,6$, $12,24,48,72 \mathrm{~h}$ ). (A) The expression of CHOP mRNA levels was examined by reverse transcription-quantitative polymerase chain reaction. $\beta$-actin served as an internal control. (B) The expression of $\mathrm{CHOP}$ at the protein level was examined by western blot analysis. $\beta$-actin served as a loading control. ${ }^{*} \mathrm{P}<0.05$ vs. $0 \mathrm{~h}, \mathrm{n}=5$.

that tunicamycin led to significant cell injury in cardiomyocytes in a time- and dose-dependent manner.

Tunicamycin-induced cardiomyocyte injury manifested as apparent apoptosis. To assess the feature of cell injury induced by tunicamycin, a flow cytometry assay and analysis of caspase-3 activity were used to detect apoptosis. Compared with the control group, treatment with $100 \mathrm{ng} / \mathrm{ml}$ tunicamycin for $24 \mathrm{~h}$ did not induce marked apoptosis, as observed from flow cytometry assays (Fig. 2A) and activity of caspase-3 (Fig. 2B), whereas treatment with $100 \mathrm{ng} / \mathrm{ml}$ tunicamycin for 48-96 h significantly increased apoptosis (Fig. 2A), and activity of caspase-3 (Fig. 2B), particularly for $72 \mathrm{~h}$.

Tunicamycin induces upregulation of ER chaperone GRP78 in cardiomyocytes. To confirm that tunicamycin induces ER stress, endoplasmic reticulum chaperone GRP78 was assessed by RT-qPCR and western blot analysis. As shown in Fig. 3A and B, there was a relatively low level of GRP78 in normal cardiomyocytes. However, treatment with tunicamycin $(100 \mathrm{ng} / \mathrm{ml})$ upregulated GRP78 at the mRNA and protein levels. The mRNA and protein levels of GRP78 began to increase following $6 \mathrm{~h}$ exposure to tunicamycin, it was then markedly upregulated and reached the maximum at
$24 \mathrm{~h}$. Subsequently, its expression significantly declined and returned to the basal level at $72 \mathrm{~h}$. These results indicate that exposure of cardiomyocytes to tunicamycin induces upregulation of the ER resident molecular chaperone GRP78 at the mRNA and protein levels, and induces ER stress.

Tunicamycin induces CHOP expression in cardiomyocytes. To elucidate that tunicamycin induces the expression of CHOP in cardiomyocytes, CHOP levels were assessed by RT-qPCR and western blot analysis. As shown in Fig. 4A and B, there was relatively low expression of $\mathrm{CHOP}$ in normal cardiomyocytes. However, treatment with tunicamycin $(100 \mathrm{ng} / \mathrm{ml})$ increased $\mathrm{CHOP}$ at the mRNA and protein levels. $\mathrm{CHOP}$ levels began to increase at $6 \mathrm{~h}$ and reached a peak $24 \mathrm{~h}$ after exposure to $100 \mathrm{ng} / \mathrm{ml}$ tunicamycin. Subsequently, its expression slowly declined. The results provide evidence that tunicamycin triggers induction of CHOP in primary cultured neonatal rat cardiomyocytes.

\section{Discussion}

In the present study, an endoplasmic reticulum stress-induced apoptotic model was established in primary cultured neonatal rat cardiomyocytes. Firstly, it was demonstrated that 
tunicamycin resulted in cardiomyocyte injury in a time- and dose-dependent manner. Secondly, apoptosis was the predominant mode of cell death of cardiomyocytes induced by tunicamycin. Thirdly, tunicamycin upregulated a number of ER stress markers, such as the survival/rescue protein GRP78 and the apoptotic molecule CHOP.

Accumulating evidencedemonstrates thatER stress-induced apoptosis is the key contributor to cell loss in the pathogenesis of a series of cardiovascular diseases $(8,25,26)$. However, the detailed mechanism of ER stress in cardiovascular diseases remains unclear. Tunicamycin is a specific inhibitor of $\mathrm{N}$-linked glycosylation of protein, which is observed only in the endoplasmic reticulum, showing that tunicamycin is a highly specific ER stress inducer. Therefore, the present study aimed to establish an ER stress-induced apoptotic model using tunicamycin in primary cultured neonatal rat cardiomyocytes. Although tunicamycin has been used to induce ER stress in other cell types, cells are often regulated in a typeand stimulus-specific manner $(27,28)$. Therefore, in order to screen the optimal treatment time and concentration of ER stress-induced cell injury by tunicamycin in cardiomyocytes, cell viability was assessed by an MTT assay, and cardiomyocyte injury was detected using an LDH release assay.

However, the pattern of cardiomyocyte injury-induced by tunicamycin remains unclear. Flow cytometry and analysis of the activity of caspase- 3 were conducted to quantify the number of apoptotic cells. The present data revealed that tunicamycin induced cardiomycyte apoptosis. In addition, treatment of primary cultured neonatal rat cardiomyocytes with $100 \mathrm{ng} / \mathrm{ml}$ tunicamycin for $72 \mathrm{~h}$ led to the maximum rate of apoptosis.

Next, the effect of $100 \mathrm{ng} / \mathrm{ml}$ tunicamycin on ER stress-related molecules in cardiomyocytes was investigated. RT-qPCR and western blot analysis revealed that tunicamycin increased GRP78 and CHOP levels, and induced ER stress in primary cultured neonatal rat cardiomyocytes, which is consistent with the results of a previous study (29). GRP78 survival/rescue protein of cardiomyocytes treated with $100 \mathrm{ng} / \mathrm{ml}$ tunicamycin was rapidly upregulated, peaked at $24 \mathrm{~h}$, and then rapidly declined to near preinduction level at the mRNA and protein levels. In addition, the level of apoptotic ER stress-related molecule CHOP was low at the early phase, peaked $24 \mathrm{~h}$ following treatment with $100 \mathrm{ng} / \mathrm{ml}$ tunicamycin and then decreased slowly. These data indicated that GRP78 was rapidly upregulated in the early stages of tunicamycin-induced ER stress in cardiomyocytes, whereas CHOP gradually increased in the first $24 \mathrm{~h}$. The levels of GRP78 then rapidly decreased, whereas CHOP was slowly downregulated by persistent tunicamycin-induced ER stress in cardiomyocytes. However, how ER stress integrates its cytoprotective and proapoptotic outputs to select between life or death cell fates remains unknown.

In conclusion, the present study successfully constructed an ERS-induced cardiomyocyte apoptotic model with tunicamycin, and screened the optimal concentration and processing time for detecting cell viability, apoptotic index and ERS associated proteins GRP78 and CHOP. The results of the present study provide important experimental data for preclinical and clinical investigations of ERS-associated cardiovascular diseases.

\section{Acknowledgements}

This study was supported by the National Natural Science Foundation of China (grant nos. 81070127 and 81270169). The authors would like to thank Dr Xinliang Ma (Department of Emergency Medicine, Thomas Jefferson University, Philadelphia, USA) and Dr Jun Ren (Center for Cardiovascular Research and Alternative Medicine, University of Wyoming College of Health Sciences, Laramie, WY, USA) for commenting on the manuscript.

\section{References}

1. Millott R, Dudek E and Michalak M: The endoplasmic reticulum in cardiovascular health and disease. Can J Physiol Pharmacol 90: 1209-1217, 2012.

2. Peralta $\mathrm{C}$ and Brenner $\mathrm{C}$ : Endoplasmic reticulum stress inhibition enhances liver tolerance to ischemia/reperfusion. Curr Med Chem 18: 2016-2024, 2011.

3. Ding W, Zhang X, Huang H, Ding N, Zhang S, Hutchinson SZ and Zhang $\mathrm{X}$ : Adiponectin protects rat myocardium against chronic intermittent hypoxia-induced injury via inhibition of endoplasmic reticulum stress. PLoS One 9: e94545, 2014.

4. Gomes-Alves P, Couto F, Pesquita C, Coelho AV and Penque D: Rescue of F508del-CFTR by RXR motif inactivation triggers proteome modulation associated with the unfolded protein response. Biochim Biophys Acta 1804: 856-865, 2010.

5. Farrukh MR, Nissar UA, Afnan Q, et al: Oxidative stress mediated $\mathrm{Ca}(2+)$ release manifests endoplasmic reticulum stress leading to unfolded protein response in UV-B irradiated human skin cells. J Dermatol Sci 75: 24-35, 2014.

6. Zhang K and Kaufman RJ: From endoplasmic-reticulum stress to the inflammatory response. Nature 454: 455-462, 2008.

7. Fu HY, Okada K, Liao Y, et al: Ablation of C/EBP homologous protein attenuates endoplasmic reticulum-mediated apoptosis and cardiac dysfunction induced by pressure overload. Circulation 122: 361-369, 2010.

8. Yang L, Zhao D, Ren J and Yang J: Endoplasmic reticulum stress and protein quality control in diabetic cardiomyopathy. Biochim Biophys Acta 1852: 209-218, 2014.

9. Groenendyk J, Sreenivasaiah PK, Kim do H, Agellon LB and Michalak M: Biology of endoplasmic reticulum stress in the heart. Circ Res 107: 1185-1197, 2010.

10. Avery J, Etzion S, DeBosch BJ, et al: TRB3 function in cardiac endoplasmic reticulum stress. Circ Res 106: 1516-1523, 2010.

11. Liu D, Zhang M and Yin H: Signaling pathways involved in endoplasmic reticulum stress-induced neuronal apoptosis. Int J Neurosci 123: 155-162, 2013.

12. Thuerauf DJ, Marcinko M, Gude N, Rubio M, Sussman MA and Glembotski CC: Activation of the unfolded protein response in infarcted mouse heart and hypoxic cultured cardiac myocytes. Circ Res 99: 275-282, 2006.

13. Endo H, Murata K, Mukai M, Ishikawa $\mathrm{O}$ and Inoue $\mathrm{M}$ : Activation of insulin-like growth factor signaling induces apoptotic cell death under prolonged hypoxia by enhancing endoplasmic reticulum stress response. Cancer Res 67: 8095-8103, 2007.

14. Erbay E, Babaev VR, Mayers JR, et al: Reducing endoplasmic reticulum stress through a macrophage lipid chaperone alleviates atherosclerosis. Nat Med 15: 1383-1391, 2009.

15. Hotamisligil GS: Endoplasmic reticulum stress and atherosclerosis. Nat Med 16: 396-399, 2010.

16. Myoishi M, Hao H, Minamino T, et al: Increased endoplasmic reticulum stress in atherosclerotic plaques associated with acute coronary syndrome. Circulation 116: 1226-1233, 2007.

17. Glembotski CC: Endoplasmic reticulum stress in the heart. Circ Res 101: 975-984, 2007.

18. Kim I, Xu W and Reed JC: Cell death and endoplasmic reticulum stress: disease relevance and therapeutic opportunities. Nat Rev Drug Discov 7: 1013-1030, 2008.

19. Dickhout JG, Carlisle RE and Austin RC: Interrelationship between cardiac hypertrophy, heart failure and chronic kidney disease: endoplasmic reticulum stress as a mediator of pathogenesis. Circ Res 108: 629-642, 2011.

20. van Empel VP, Bertrand AT, Hofstra L, Crijns HJ, Doevendans PA and De Windt LJ: Myocyte apoptosis in heart failure. Cardiovasc Res 67: 21-29, 2005. 
21. Shen M, Wang L, Yang G, et al: Baicalin protects the cardiomyocytes from ER stress-induced apoptosis: inhibition of CHOP through induction of endothelial nitric oxide synthase. PLoS One 9: e88389, 2014.

22. Liu B, Niu L, Shen MZ, et al: Decreased astroglial monocarboxylate transporter 4 expression in temporal lobe epilepsy. Mol Neurobiol 50: 327-338, 2014.

23. Fu HY, Minamino T, Tsukamoto O, et al: Overexpression of endoplasmic reticulum-resident chaperone attenuates cardiomyocyte death induced by proteasome inhibition. Cardiovasc Res 79: 600-610, 2008.

24. Liu B, Wang L, Shen LL, et al: RNAi-mediated inhibition of presenilin 2 inhibits glioma cell growth and invasion and is involved in the regulation of $\mathrm{Nrg} 1 / \mathrm{ErbB}$ signaling. Neuro Oncol 14: 994-1006, 2012.

25. Zhou S, Yin X, Zheng Y, et al: Metallothionein prevents intermittent hypoxia-induced cardiac endoplasmic reticulum stress and cell death likely via activation of Akt signaling pathway in mice. Toxicol Lett 227: 113-123, 2014.
26. Yang Y, Li C, Xiang X, et al: Ursolic acid prevents endoplasmic reticulum stress-mediated apoptosis induced by heat stress in mouse cardiac myocytes. J Mol Cell Cardiol 67: 103-111, 2014.

27. Qi X, Vallentin A, Churchill E and Mochly-Rosen D: DeltaPKC participates in the endoplasmic reticulum stress-induced response in cultured cardiac myocytes and ischemic heart. J Mol Cell Cardiol 43: 420-428, 2007.

28. Okada K, Minamino T, Tsukamoto Y, et al: Prolonged endoplasmic reticulum stress in hypertrophic and failing heart after aortic constriction: possible contribution of endoplasmic reticulum stress to cardiac myocyte apoptosis. Circulation 110: 705-712, 2004.

29. Lin JH, Li H, Yasumura D, et al: IRE1 signaling affects cell fate during the unfolded protein response. Science 318: 944-949, 2007. 